\title{
Limitations on suprathermal tails of electrons in the lower solar corona
}

\author{
Y.-K. Koํㅣ L. A. Fisk \\ Department of Atmospheric, Oceanic, and Space Sciences, University of Michigan, Ann Arbor \\ G. Gloeckler ${ }^{2}$ \\ Department of Physics and ISTP, University of Maryland, College Park
}

\section{J. Geiss}

International Space Science Institute, Bern, Switzerland

\begin{abstract}
Measurements of the charge states of solar wind ions by the SWICS instrument on Ulysses are used to place limits on the extent to which the velocity distribution of electrons in the lower solar corona deviates from a Maxwellian distribution by having a suprathermal tail. It is found that the data are consistent with a suprathermal tail which is not large, and which has a minor influence on the location and magnitude of the maximum of the electron temperature. This result has implications for theories which propose that suprathermal tails of electrons in the solar corona can have a strong influence on the formation of the solar corona and the dynamics of the solar wind.
\end{abstract}

\section{Introduction}

The SWICS instrument on Ulysses [Gloeckler et al., 1992] has returned detailed information on the charge states of solar wind ions from the steady high speed stream that originates from the south polar coronal hole. These charge states are established, or "frozen-in" as the ions leave the lower solar corona, within $\sim 5$ solar radii of the Sun. The charge states can thus be used to place constraints on conditions in the lower corona, such as the electron temperature and density, and the flow speed of the ions. It is the purpose of this paper to use the observed charge state data to place limits on the extent to which the velocity distribution of the electrons in the lower corona can have a significant suprathermal tail. Such tails have been argued by Scudder [1992a,b] to play a significant role in the deposition of energy and thus in the formation of the corona, in the formation of Doppler line widths, and in the dynamics of the solar wind acceleration. We find that the charge state data are most consistent with a velocity distribution for the electrons, within 5 solar radii of the Sun, with a suprathermal tail which is not large. In the nomenclature for describing suprathermal tails, the $\kappa$ value for the non-Maxwellian distribution must be greater than 5 . Such a tail has minor influence on either the magnitude or the location of the temperature maximum for coronal electrons.

\footnotetext{
${ }^{1}$ Visiting at Department of Physics, University of Maryland, College Park.

${ }^{2}$ Also Department of Atmospheric, Oceanic, and Space Sciences, University of Michigan, Ann Arbor.
}

Copyright 1996 by the American Geophysical Union.

Paper number 96GL02449.

0094-8534/96/96GL-02449\$05.00
We begin by describing the technique that we use to constrain the suprathermal tail. This technique is described in more detail in Ko et al. [1996] ('An empirical study of the electron temperature profile and heavy ion velocities in the south polar coronal hole', submitted to the Solar Physics) and applied to determine other aspects of the behavior of coronal electrons and heavy ions. We then present the evidence that the suprathermal tail of coronal electrons is not large. Concluding remarks are provided in the final section.

\section{The Technique}

As the heavy ions are convected outward through the solar corona with the expanding solar wind, their charge states will evolve through both ionization and recombination. For a steady solar wind, then, the ionic fraction, $y_{i}$, of a given charge state, which is defined as the ratio of the number density of an ion with charge $+i$ to the total number density of all ion states of this element, will evolve subject to the equation: [Hundhausen, Gilbert, and Bame 1968]

$$
u \frac{d y_{t}}{d r}=n_{e}\left(y_{t-1} C_{i-1}-y_{i}\left(C_{t}+R_{t-1}\right)+y_{t+1} R_{i}\right)
$$

Here, $C_{i}$ is the total ionization rate (including collisional ionization and autoionization) from charge $+\mathrm{i}$ to $+\mathrm{i}+1$ and $R$ is the total recombination rate (including radiative recombination and dielectronic recombination) from charge $+i+1$ to $+i$. The ionization rates and recombination rates used here are the same as those adopted in Ko et al. [1996]. We assume that all ions of the same element have the same speed $u$ and flow only in the radial direction; $n_{e}$ is the electron number density. Note that the sum of all $y_{i}$ 's for a given element must be unity.

At some heliocentric radial distance, typically $<5$ solar radii, the ionic fractions will cease to change [e.g. Owocki $e t$ $a l ., 1983$; Bürgi and Geiss, 1986]. The electron density falls off with radial distance; the ion speeds increase; and from (1), $y_{i}$ becomes constant. At this distance the ionic fractions are said to be frozen-in, and it is these ionic fractions that are observed by Ulysses at several AU from the Sun.

The ionization and recombination rates are functions of the electron temperature $T_{e}$, and will depend on whether the electron distribution is Maxwellian. Thus, the ionic fraction that will be frozen-in depends on $T_{e}(r), n_{e}(r), u(r)$, and the shape of the electron distribution. Equivalently, the observed ionic fractions can be used to place constraints on allowable values for and radial dependencies of $T_{e}, n_{e}, u$, and the shape of the electron distribution. 


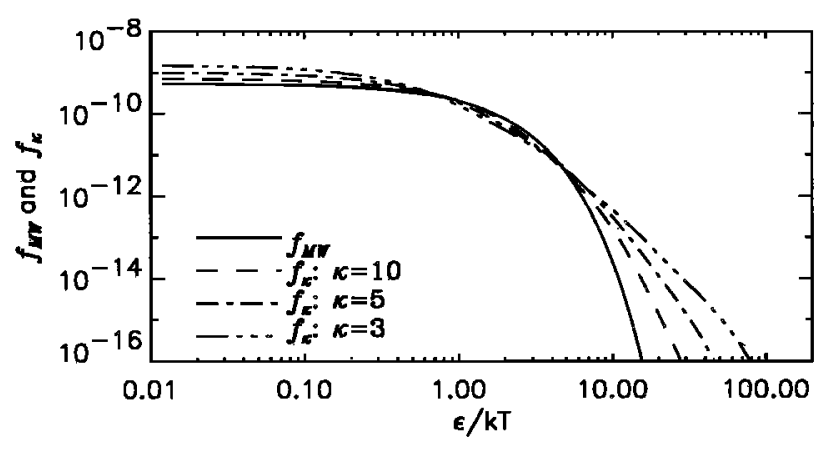

Figure 1. The electron distribution function with $\kappa=3$ (dashdot-dot-dot curve), $x=5$ (dash-dot curve) and $\kappa=10$ (dashed curve) compared with the Maxwellian distribution function (solid curve) at the same electron temperature of $1.5 \times 10^{6}{ }^{\circ} \mathrm{K}$.

We concentrate here on determining the shape of the electron distribution, i. e. the extent to which a suprathermal tail is permitted. To do so, we use a technique which is described in detail in Ko et al. [1996]. We use observations to determine $n_{e}$, and we develop parametric descriptions for $T_{e}, u$, and of the shape of the electron distribution. We then do a systematic search to determine the values of the parameters that yield the best fit to the charge states observed by Ulysses. In particular, the following sequence of operations is used:

1. The electron density profile in the south polar coronal hole has been derived from observations by the White Light Coronagraph on the Spartan 201-01 mission [Fisher and Guhathakurta, 1995]. These observations were made one year earlier than the SWICS observations of the solar wind charge states from this coronal hole. Nonetheless, all the observations are near solar minimum when significant time variations are not expected. We thus consider $n_{e}(r)$ to be determined by the observed Spartan profile.

2. The SWICS/Ulysses data have indicated that the electron temperature profile in the south polar coronal hole has a local temperature maximum with $T_{\max } \approx 1.55 \times 10^{6}{ }^{\circ} \mathrm{K}$ [Geiss et al., 1995; Ko et al., 1996]. We thus take the electron temperature profile to have the following form:

For $r<R_{\max }$,

$$
T_{e}(r)=T_{\max } /\left(1+\left(R_{\max }-r\right)^{f}\right)^{x}
$$

For $r>R_{\max }$,

$$
T_{e}(r)=T_{\max } /\left(1+\left(r-R_{\max }\right)^{2}\right)^{y}
$$

Where $\quad x=\log \left[T_{\max } / T_{1}\right] / \log \left[1+\left(R_{\max }-1\right)^{f}\right]$ $y=\log 2 / \log \left[1+R_{\text {wid }}^{2}\right]$. Here, $R_{\max }\left(\right.$ in solar radii $R_{o}$ ) is the location of the temperature maximum, $T_{\max }$. The parameters $f$ and $R_{w t d}$ determine the shape of the temperature profile; $T_{1}$ is the temperature at one solar radii. As is discussed in Ko et al. [1996], the parameters to which the charge states are most sensitive are $T_{\max }$ and $R_{\max } ; T_{1}$ must be chosen to be < $7.5 \times 10^{5}{ }^{\circ} \mathrm{K}$.

3. The speeds of the heavy ions in the lower corona are expected to be different than that of the protons and electrons. The effects of inertia and compensating Coulomb drag could result in the heavy ions flowing slower than the protons [e.g. Bürgi and Geiss, 1986]; wave effects could conceivably cause the heavy ions to flow faster. We take the speed profile, in $\mathrm{km} / \mathrm{s}$, of all the ions of a given element to be:

$$
u(r)=699[(r-1) / 9]^{p}+1
$$

The speed is then $1 \mathrm{~km} / \mathrm{s}$ at $1 R_{o}$ and $700 \mathrm{~km} / \mathrm{s}$ at $10 R_{o}$ which should cover all possible values of the ion velocities within $10 R_{o}$. However, the speed profile can vary depending on the parameter $p$.

4. Electron distributions that have a strong suprathermal tail are frequently described by the so-called kappa distribution which was introduced by Olbert [1969]:

$$
f_{\kappa}(\varepsilon)=\left(\frac{m}{2 \pi k T}\right)^{3 / 2} A_{\kappa}\left[1+\frac{\varepsilon}{(\kappa-1.5) k T}\right]^{-\kappa-1}
$$

where $A_{\kappa}=\Gamma(\kappa+1) /\left[\Gamma(\kappa-0.5)(\kappa-1.5)^{1.5}\right]$ and $\varepsilon=m v^{2} / 2$; $m$ is particle mass, $v$ is particle speed, and $k$ is Boltzmann's constant. The kappa distribution resembles a Gaussian distribution at low particle speeds and evolves smoothly into an inverse power law with increasing $v$. The normalization is such that integrating over all speeds yields unity and the average value of $\varepsilon$ is $3 k T / 2$. For large values of $\kappa(\kappa>10)$ the kappa distribution approaches a simple Maxwellian; for small values of $\kappa(\kappa<4)$ the suprathermal tail is very strong. A kappa distribution has been incorporated into the calculation of the ionization and recombination rates for various ions in Owocki and Scudder [1983], Bürgi [1987] and Dzifcáková [1992]. In this work, we simulate the kappa distribution by superimposing three Maxwellian distributions for $\kappa \geq 7$ (and five Maxwellian distributions for $\kappa<7$ ) with different temperatures and normalizations. This 'simulated' kappa function yields a reasonable fit (within 15\%) to the 'real' kappa distribution (eq.(5)). The ionization and recombination rates are then the sum of those for each of the three (or five) Maxwellian distributions. The uncertainty of $15 \%$ in our fit to the kappa distribution is less than the uncertainty in the atomic rates. Figure 1 shows these 'simulated' kappa functions with $\kappa=3,5,10$ and compares them with the Maxwellian distribution function.

5. With the observed profile for $n_{e}(r)$ and by choosing the parameters to specify $T_{e}(r)$ and $u(r)$, and the equivalent $K$ value, we use (1) to determine the frozen-in charge states for
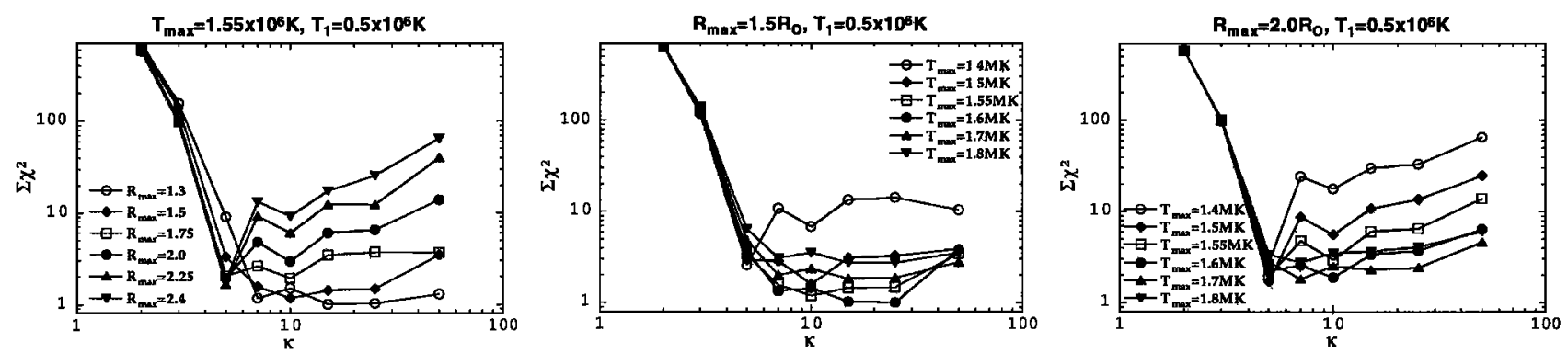

Figure 2. $\Sigma \chi^{2}$ versus $\kappa$ for various electron temperature profiles. In general, $5 \leq \kappa \leq 10$ gives better fit with the data. 

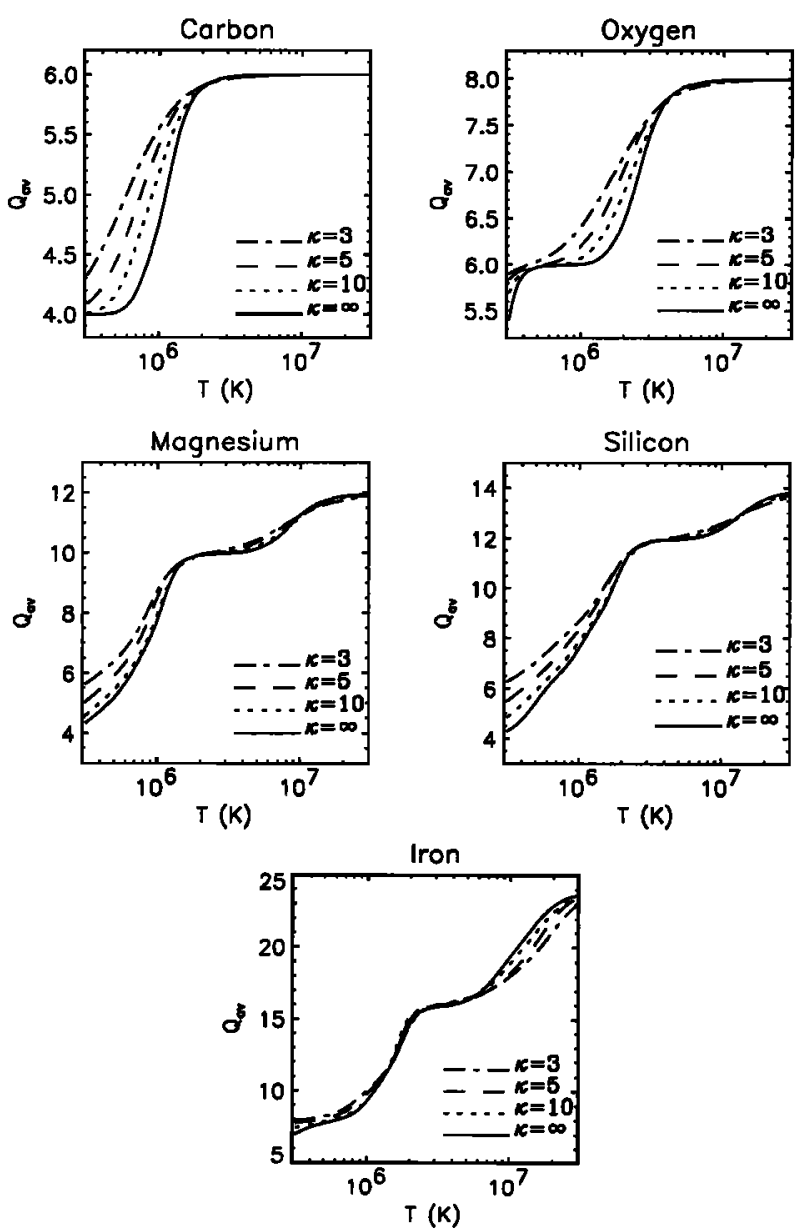

Figure 3. The mean charge states as a function of electron temperature at various values of $\kappa$ for $\mathrm{C}, \mathrm{O}, \mathrm{Mg}, \mathrm{Si}$, and $\mathrm{Fe}$ ions. $\mathrm{C}$ and $\mathrm{O}$ ions are most affected by the suprathermal tail of electron distribution at coronal temperatures.

carbon, oxygen, magnesium, silicon and iron, and then compare these results with the observations from the SWICS instrument on Ulysses, as it observed the solar wind from the south polar coronal hole. SWICS measures the intensity of solar wind and suprathermal ions as a function of their energy per charge $(E / \mathrm{q})$, mass $(\mathrm{m})$, and charge state $(\mathrm{q})$, from 0.6 to $60 \mathrm{keV} / \mathrm{e}$ in logarithmically spaced steps with $\Delta E / E \approx 0.04$ [Gloeckler et al., 1992]. Ulysses passed downward in solar latitude from the solar equatorial plane, and at latitudes above about 35 degrees it was continuously in the steady high speed stream from the south polar coronal hole. We use data from SWICS that are averaged from day 170 to 270 in 1994 , when the flow speed was nearly constant at about $780 \mathrm{~km} / \mathrm{s}$. To compare the model predictions and observation quantitatively we define a reduced $\chi$-square for each element, or

$$
\chi_{r}^{2}=\sum_{i=1}^{l} \frac{1}{l}\left(\frac{y_{i, \text { data }}-y_{i, \text { model }}}{\varepsilon_{l}}\right)^{2}
$$

where $y_{i, d a t a}$ is the measured ionic fraction, $y_{i, \text { model }}$ is the predicted ionic fraction, $\varepsilon_{i}$ is the error in $y_{t, \text { data }}$, and $l$ is the number of charge states considered. Clearly, if $\chi_{r}^{2}<1$ the model results deviate, on average, from the data by less than one error bar.

The procedure then is straight-forward. We use the observed profile for $n_{e}(r)$; we consider a likely range of values for the parameters which describe $T_{e}(r)$; and we consider a range of possible values for $\kappa$. For each choice of these parameters we then vary the parameter $p$ which describes $u(r)$ in (4) and determine the minimum value of $\chi_{r}^{2}$ for each element and record the sum of these minimum values, or $\Sigma \chi^{2}$, for all five elements. The choices for the parameters describing $T_{e}(r)$ and the values of $\kappa$ that yield the smallest values of $\Sigma \chi^{2}$ are the most likely choices for the actual coronal conditions.

We then perform this procedure in reverse. We take the most likely value of $\kappa$ and then do a full parameter search to find the most likely value of the temperature maximum and the location of the temperature maximum, for comparison with the results in which the electron distribution is Maxwellian only.

\section{The Results}

We take $f=1, R_{\text {wid }}=2 R_{o}$, and $T_{1}=5 \times 10^{5}{ }^{\circ} \mathrm{K}$ in (2) and (3) (from Ko et al. [1996], the predicted charge states are not sensitive to $f$ and $R_{\text {wid }}$, and $T_{1}$ is within the allowable range to fit the observed charge state data). We then vary $T_{\max }$ and $R_{\text {max }}$ in the range $1.3 \times 10^{6}{ }^{\circ} \mathrm{K} \leq T_{\max } \leq 1.8 \times 10^{6}{ }^{\circ} \mathrm{K} \mathrm{K}$ and $1.3 R_{o} \leq R_{\max } \leq 3.0 R_{o}$. These ranges should encompass any allowable location or value for the temperature maximum. We vary the value for kappa in the range $2 \leq \kappa \leq 25$. This range is broad and describes electron distribution functions which have strong suprathermal tails (small $\kappa$ ) through distribution functions that are indistinguishable from a Maxwellian distribution (large $\kappa$ ). Following our technique, then, we vary $p$ in (4) for each different element over the range $0.01 \leq p \leq 20$, which should allow a full range of possible speed profiles. Finally we determine $\Sigma \chi^{2}$.

The results are shown in Figure 2. In the left panel $\Sigma \chi^{2}$ is plotted versus $\kappa$ for a specific choice of $T_{\max }$ and a range of possible $R_{\max }$. In the center and right panels, $R_{\max }$ is fixed and $\Sigma \chi^{2}$ is plotted versus $\kappa$ for various choices of $T_{\max }$. In all cases, the minimum value of $\Sigma \chi^{2}$ and thus the best fit to the observed charge states occurs for larger values of $\boldsymbol{K}$ ( $\kappa \geq 5$ ). Indeed, only in the cases of the larger values of $R_{\max }$ does $\boldsymbol{\kappa}$ of order 5 provide a better fit. A closer examination of these cases reveals that they require that the flow speeds of $\mathrm{Fe}$ ions are larger than those of the lighter ions, which seems incompatible with ions being accelerated by Coulomb drag. In all cases, small values of $\kappa, \kappa<5$, or equivalently strong suprathermal tails, provide a demonstrably poor fit to the data. Clearly, the electron distribution that is consistent with the data (i.e. $\kappa \geq 5$ ) has a suprathermal tail which is not large (see Fig.1).

The presence of a strong suprathermal tail would have most influence on the charge states of carbon and oxygen, as is illustrated in Figure 3. Here the mean charge states for various elements in ionization equilibrium are plotted versus temperature, for various values of $\kappa$. Clearly, in the temperature ranges we consider, $\sim 10^{6}{ }^{\circ} \mathrm{K}$, the mean charges of carbon and oxygen are most sensitive to the value of $\kappa$. Since carbon and oxygen ions freeze-in before the temperature maximum due to their small ionization rates, a strong suprathermal tail could occur only if the coronal temperature is unreasonably low $\left(T<2 \times 10^{5}{ }^{\circ} \mathrm{K}\right)$ and the ion velocities are unreasonably high near the coronal base. Thus, it is the observed charge states of carbon and oxygen which preclude a strong suprathermal tail in the lower corona.

Consider, then, the problem in reverse. We fix $\kappa$ at 10 and do a full parameter search, i.e. we vary $T_{\max }, R_{\max }, R_{\text {wid }}, f$, $T_{1}$, and $p$, and find the best fit to the observed charge states. We first eliminate any fit for which $\Sigma \chi^{2}>5$. We then bin all 

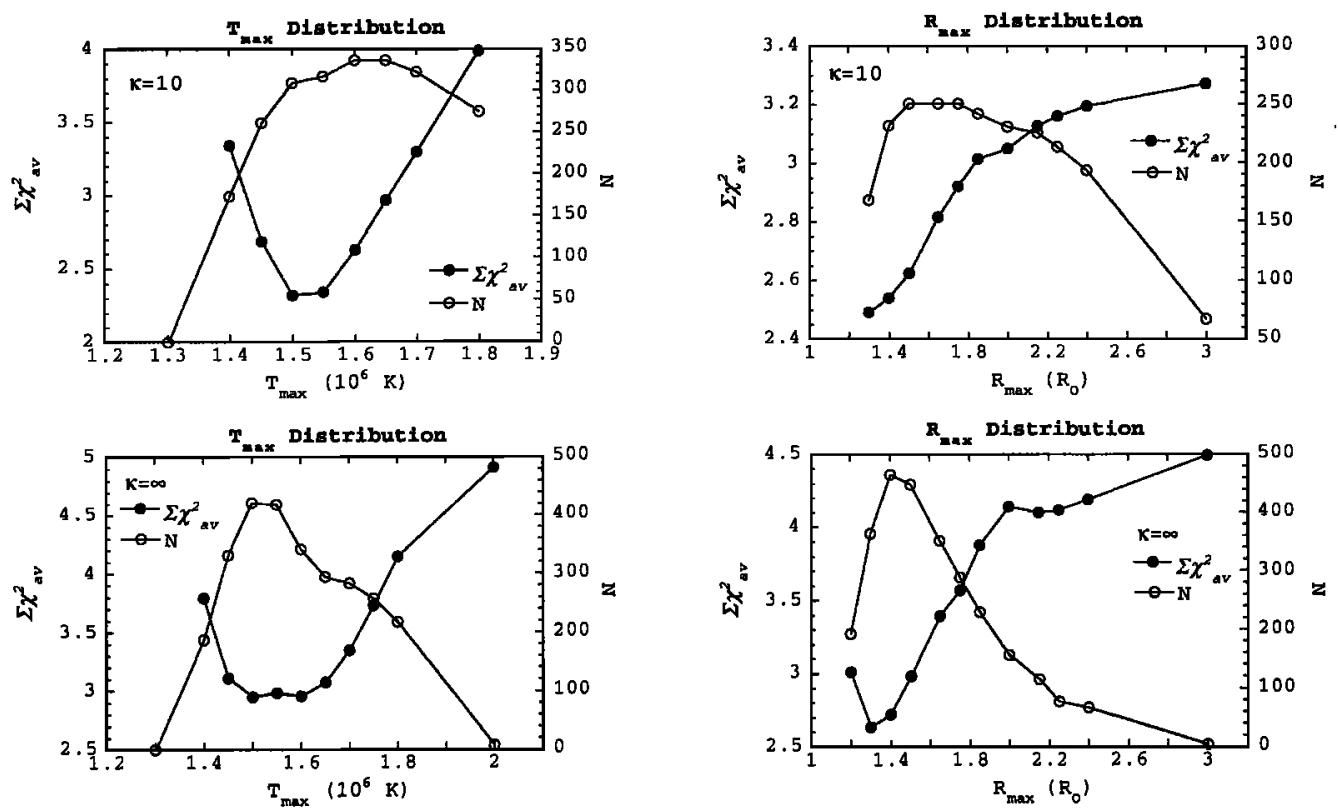

Figure 4. Upper panels: $T_{\max }$ and $R_{\max }$ distribution for a non-thermal distribution of electrons with $\kappa=10$. Lower panels: same but for Maxwellian distribution of electrons $(K=\infty)$. Note that the smallest $\Sigma \chi^{2}{ }_{a v}$ with the largest $\mathrm{N}$ provides the best fit to the observed charge states.

of the values of $\Sigma \chi^{2}$ for different values of $T_{\max }$ and $R_{\max }$, and determine the average value of $\Sigma \chi^{2}$, or $\Sigma \chi_{a v}^{2}$, in each bin. The results are shown in the Figure 4, where we plot $\Sigma \chi_{a v}^{2}$ and the number of samples, $\mathrm{N}$, in each bin versus $T_{\max }$ and $R_{\max }$. The smallest $\Sigma \chi^{2}{ }_{a \nu}$ with the largest $\mathrm{N}$ provides the best fit to the observed charge states. Clearly, the best-fit temperature maximum value is $\sim 1.55 \times 10^{6}{ }^{\circ} \mathrm{K}$. The location of the maximum is $\sim 1.4 R_{o}$. Shown in the lower panels of Figure 4 are the results of the same parameter search for an electron distribution function that is a Maxwellian. Although there are some small shifts in the value of $T_{\max }$ and $R_{\max }$, it is clear that an electron distribution with the inferred value of $\kappa=10$ has little effect on the location or the magnitude of the electron temperature maximum in the lower corona. If we take $\kappa=5$, the temperature maximum exhibits little variations $\left(T_{\max }\right.$ is lower by $10^{5}{ }^{\circ} \mathrm{K}$ ) from that results from a Maxwellian; however, $R_{\max }$ would be larger by $0.5 R_{o}$. Recall that this latter case can occur only if it is acceptable for $\mathrm{Fe}$ ions to move faster than lighter ions.

\section{Concluding Remarks}

We have performed a parameter search of possible values for the electron temperature profile in the lower solar corona, of possible speed profiles of heavy ions, and of possible forms for the electron distribution function, and determined that the charge states of heavy ions observed by the SWICS instrument on Ulysses are most consistent with a suprathermal tail on the electron distribution which is not large $(\kappa \geq 5)$. However, it should be noted that the observed charge states are best fit when we assume some suprathermal tail, $\kappa \sim 10$, as opposed to a larger value $\kappa>25$ which is indistinguishable from a Maxwellian distribution. Such a small tail is unlikely to contribute significantly to the formation of the corona, or to the dynamics of the solar wind.

Acknowledgments. This work was supported by NASA/JPL contract 955460 .

\section{References}

Bürgi, A., Effects of non-Maxwellian electron velocity distribution functions and nonspherical geometry on minor ions in the solar wind, J. Geophys. Res., 92, 1057-1066, 1987.

Bürgi, A., and Geiss, J., Helium and minor ions in the corona and solar wind: dynamics and charge states, Solar Phys., 103, 347-383, 1986.

Dzifcáková, E., The Ionization Balance of the Fe in the Solar Corona for a Non-Maxwellian Electron Distribution Function, Solar Phys., 140, 247-267, 1992.

Fisher, R., and Guhathakurta, M., Physical properties of polar coronal rays and holes as observed with SPARTAN 201-01 Coronagraph, Astrophys. J., 447, L139-L142, 1995.

Geiss, J. et al. The Southern High Speed Stream: Results from SWICS/Ulysses, Science, 268, 1033-1036, 1995.

Gloeckler, G. et al., The solar wind ion composition spectrometer, Astron. Astrophys. Suppl. Ser., 92, 267-289, 1992.

Hundhausen, A. J., Gilbert, H. E., and Bame, S. J., The State of Ionization of Oxygen in the Solar Wind, Astrophys. J., 152, L3-L5, 1968.

Olbert, S., Summary of experimental results from M.I.T. detector on IMP-1, in Physics of the Magnetospheres, edited by R. L. Carovillano, J. F. McClay, and H. R. Radoski, pp.641-659, D. Reidel, Hingham, Mass., 1969.

Owocki, S. P., Holzer, T. E., and Hundhausen, A. J., The solar wind ionization state as a coronal temperature diagnostic, Astrophys. J., $275,354-366,1983$.

Owocki, S. P., and Scudder, J. D., The effect of a non-Maxwellian electron distribution on oxygen and iron ionization balances in the solar corona, Astrophys. J., 270, 758-768, 1983.

Scudder, J. D., On the causes of temperature change in inhomogeneous low-density astrophysical plasmas, Astrophys. J., 398, 299-318, $1992 a$.

Scudder, J. D., Why all stars should possess circumstellar temperature inversions, Astrophys. J., 398, 319-349, 1992b.

Y.-K. Ko, Space Physics Group, University of Maryland, College Park, MD 20742 (e-mail: ko@umdsp.umd.edu).

L. A. Fisk, Department of Atmospheric, Oceanic and Space Sciences, University of Michigan, Ann Arbor, MI 48105 (e-mail: LA_Fisk@emal.sprl.umich.edu).

G. Gloeckler, Space Physics Group, University of Maryland, College Park, MD 20742(e-mail: gloeckler@umdsp.umd.edu).

J. Geiss, International Space Science Institute, Hallerstrasse 6, CH 3012, Bern, Switzerland.

(received: April 23, 1996; revised: June 26, 1996;

accepted: July 25,1996 .) 